\title{
Depressão na adolescência: características clínicas
}

\author{
Saint-Clair Bahls \\ Flávia Rocha Campos Bahls \\ Universidade Federal do Paraná
}

\begin{abstract}
Resumo
Atualmente, a depressão em adolescentes é considerada comum, debilitante e recorrente, envolvendo um alto grau de morbidade e mortalidade, representando um sério problema de saúde pública, mesmo sendo bastante recente o interesse científico pela depressão neste período da vida. Os sistemas diagnósticos atuais definem que os sintomas básicos de um episódio depressivo maior são os mesmos em adolescentes e adultos, entretanto, os pesquisadores destacam a importância do processo de maturação na apresentação sintomatológica da depressão, com características predominantes em cada fase do desenvolvimento. Este artigo visa apresentar uma revisão sobre as características clínicas da depressão na adolescência com o objetivo de contribuir com o esclarecimento desta patologia grave, comum e ainda pouco reconhecida.

Palavras-chave: depressão; adolescência; características clínicas.
\end{abstract}

\begin{abstract}
Depression in adolescence: clinical features

Nowadays, depression in adolescents is understood to be a common, disabling and recurrent disease, with a high level of morbidity and mortality. Thus, it is an important public health problem. Nevertheless, the scientific concern with depression in adolescence is recent. The present diagnostic systems define that the basic features are the same in adolescents and adults in a major depression episode; however, researchers emphasize the importance of the developmental process in the clinical manifestations, with predominant features in each phase. This paper reviews the clinical features in depressed adolescents contributing to a better understanding of this common, severe and not very well recognized pathology.

Keywords: depression; adolescence; clinical features.
\end{abstract}

Os transtornos depressivos apresentam alta e crescente prevalência na população geral (Bahls, 1999), e existem evidências científicas suficientes situando as depressões entre as doenças mais comuns, prejudiciais e que causam mais custos sociais, representando um problema dos mais graves em saúde pública, com impacto em todos os níveis da sociedade (Judd, 1995). A Organização Mundial de Saúde indica que nas próximas duas décadas haverá uma mudança nas necessidades de saúde da população mundial devido ao fato de que doenças como a depressão estão, rapidamente, substituindo os tradicionais problemas das doenças infecciosas e de má nutrição. O prejuízo causado pelas doenças, medido pela Disability Adjusted Life Years, mostra que a depressão maior, quarta causa geradora de sobrecarga em 1990, será a segunda causa no ano 2020, só perdendo para doenças cardíacas isquêmicas (Bahls, 1999; Murray \& Lopez, 1996). Especialmente se considerarmos que o curso clínico da depressão maior costuma ser marcado por recorrências e cronicidade (Hirschfeld, 1998), podemos estabelecer uma noção clara da importância e gravidade desta patologia na população geral.

Entretanto, o interesse científico pela depressão em adolescentes é bastante recente, pois, até a década de 70, acreditava-se que a depressão nessa faixa etária fosse rara (Bahls, 2002; Besseghini, 1997; Goodyer, 1996; Herkov \& Myers, 1996; I, Silva \& Machado, 1998; Kent, Vostanis \& Feehan, 1997; Larsson, Melin, Breitholtz \& Andersson, 1991; Scivoletto, Nicastri \& Zilberman, 1994; Shaffi \& Shaffi, 1992; Souza, 1984). Atualmente considera-se que a depressão maior na adolescência é comum, e seu reconhecimento ocorreu oficialmente pelo Instituto Nacional de Saúde Mental dos EUA (NIMH) em 1975 (Bhatara, 1992). Apresenta natureza duradoura e pervasiva, afeta múltiplas funções e causa significativos danos psicossociais. Em artigo recente, Ward, Eyler e Makris (2000) pontuam que a depressão se transformou em uma das doenças mais comumente encontradas pelos médicos que atendem adolescentes. Alguns autores afirmam que a depressão maior na adolescência é mais grave e perniciosa do 
que em adultos, e seu curso é mais refratário do que na depressão de início na idade adulta (Martin \& Cohen, 2000; Rohde, Lewinsohn \& Seeley, 1994).

A depressão em adolescentes parece estar mais freqüente e ocorrendo cada vez mais cedo (Birmaher e cols., 1996; Brent, 1993; Garrison, Addy, Jackson, Mckeown \& Waller, 1997; Kessler \& Walters, 1998; Prosser \& McArdle, 1996; Ryan e cols., 1992; Versiani, Reis \& Figueira, 2000). No estudo Los Angeles Epidemiologic Catchment Area Project, conforme Olsson e von Knorring (1999), 25\% dos adultos com depressão maior relataram o primeiro episódio da doença ocorrendo antes dos dezoito anos de idade. Estudos longitudinais com amostras comunitárias têm demonstrado um aumento secular em transtornos depressivos em adultos e também tem sido observado que o risco para transtornos depressivos é maior em pessoas nascidas mais recentemente, fenômeno chamado de "efeito de corte" em epidemiologia (Cross-National Collaborative Group, 1992; Joyce, Oakley-Browne, Wells, Buschnell \& Hornblow, 1990; Klerman e Weissman, 1989). Aumento secular significa que indivíduos nascidos na última parte do século XX têm uma tendência de início mais precoce e um maior risco para depressão (Gill, Coffey \& Park, 2000). Klerman e cols. (1985) observaram um aumento no risco de adoecer de depressão grave ao longo da vida nas pessoas nascidas nos EUA após a II Guerra Mundial. E Ryan e cols. (1992) encontraram pesquisando o risco de depressão em famílias, que as pessoas nascidas mais tarde apresentaram um maior grau de risco, e o efeito do ano de nascimento foi estatisticamente significativo. Kessler e Walters (1998) em estudo epidemiológico em cortes populacionais sucessivos do National Comorbidity Survey (EUA), relataram a presença do efeito de corte na depressão maior em adolescentes, pois seus resultados indicaram a presença de aumento secular na prevalência ao longo da vida para depressão maior entre jovens.

\section{Epidemiologia}

A prevalência de depressão maior em adolescentes, segundo artigos de revisão, variam de 0,4 a 10,0\% com claro predomínio das meninas sobre os meninos, e, em relação ao fator idade, a maioria dos artigos destacam um aumento importante na passagem da infância para a adolescência (Bahls, no prelo A).

Segundo vários autores a ocorrência de depressão aumenta significativamente durante a adolescência, havendo uma modificação na distribuição entre os sexos. Citam que em crianças não há diferença entre os gêneros, ou os meninos apresentam taxas um pouco maiores do que as meninas, entretanto, na adolescência, ocorre uma modificação com um predomínio das meninas sobre os meninos (DSM-IV, 1994; Herkov \& Myers, 1996; Scivoletto e cols., 1994; Versiani e cols., 2000; Walter, 1996). Segundo Angold e Costello (1995), dos dez aos quatorze anos de idade ocorre um aumento de três a quatro vezes nos índices de depressão; semelhante citação faz I (1996) ao referir que, a partir dos dez anos de idade, ocorre um aumento na prevalência da depressão em ambos os sexos, mais acentuado nas meninas. A partir de estudos retrospectivos, Besseguini (1997) e Lewinsohn, Clarke, Seeley e Rohde (1994) afirmam que a prevalência da depressão é baixa até a idade dos nove anos, aumentando agudamente dos nove aos dezenove anos, especialmente nas meninas. NolenHoeksema e Girgus (1994) citam que é por volta dos treze a quatorze anos que as meninas começam a apresentar taxas mais altas de depressão do que os meninos, sendo que, após os quinze anos, as meninas são duas vezes mais afetadas. Estes dados indicam, portanto, que os índices de depressão aumentam consideravelmente na adolescência, com taxas e distribuição no gênero (predomímio feminino) semelhantes aos dos adultos.

Em recente revisão sobre a epidemiologia dos transtornos depressivos em amostras comunitárias de adolescentes (Bahls, no prelo A), observou-se que os resultados e a metodologia empregada nos artigos de pesquisa apresentam muitas diferenças. Nos nove artigos revisados ocorrera o emprego de cinco diferentes tipos de entrevistas estruturadas ou semiestruturadas, com os resultados variando, para a prevalência-ano em depressão maior de 3,3 a 12,4\% e para a distimia de 2,0 a 6,4\%. Quanto à distribuição no gênero, os resultados foram conflitantes, pois, em três pesquisas, houve um predomínio das meninas sobre os meninos, enquanto em três outras não foram encontradas diferenças estatisticamente significativa e em outro estudo o predomínio foi dos meninos sobre as meninas. Quanto ao fator idade, só citado em duas das pesquisas, não foi encontrada diferença estatisticamente significativa. Portanto, os artigos de pesquisa não confirmam os dados dos artigos de revisão tanto em relação ao fator sexo quanto ao fator idade.

Os estudos epidemiológicos sobre depressão em adolescentes apresentam algumas discrepâncias nos valores das taxas encontradas e na sua distribuição por gênero. A partir da literatura, observa-se que, apesar da aceitação do aumento da prevalência da depressão durante a adolescência, não se conhece claramente em que momento dessa fase da vida isso ocorre. Também 
não se sabe em que instante se dá o aumento das taxas em relação ao gênero com o predomínio das meninas sobre os meninos, pois os dados disponíveis são ainda relativamente escassos (Bahls, 2002; Baptista \& Golfeto, 2000; Chartier \& Lassen, 1994; Roberts \& Chen, 1995).

\section{Quadro Clínico}

Adolescentes e adultos quando adoecidos de transtornos depressivos apresentam sintomatologia semelhante, que permite sua compreensão como entidades fenomenológicas iguais. Esta consideração foi derivada de várias pesquisas que definiram que os mesmos critérios diagnósticos são confiavelmente aplicados em adolescentes e em adultos (Birmaher e cols., 1996; Feijó, Saueressig, Salazar \& Chaves, 1997; Harrington, 1992; Olsson \& von Knorring, 1997a; Pataki \& Carlson, 1995; Roberts, Lewinsohn \& Seeley, 1995; Scivoletto e cols., 1994; Shaffi \& Shaffi, 1992; Walter, 1996; York \& Hill, 1997). Portanto, os critérios diagnósticos utilizados pelos atuais sistemas de classificação (Manual Diagnóstivo e Estatístico de Transtornos Mentais, DSM-IV, 1994; Classificação Internacional das Doenças, CID-10, 1992) são os mesmos, não diferenciando a adolescência de outras épocas da vida. Os principais transtornos depressivos são a depressão maior e a distimia.

Entretanto, é necessário destacar que a maioria dos autores na área dos transtornos depressivos na infância e na adolescência cita que os sintomas variam com a idade, enfatizando a importância do processo de maturação das diferentes fases do desenvolvimento dos sinais e comportamentos depressivos, existindo uma caracterização sintomatológica predominante por faixa etária (Berganza \& Aguilar, 1992; Busse, 1996; Goodyer \& Cooper, 1993; I, 1996; Kessler \& Walters, 1998; Morgan, 1994; Nolen-Hoeksema \& Girgus, 1994; Sadler, 1991; Shaffi \& Shaffi, 1992; Versiani e cols., 2000). Examinaremos a seguir as principais características da sintomatologia depressiva em adolescentes.

Em adolescentes existem importantes características fenomenológicas que são típicas do transtorno depressivo maior nesta fase da vida. Adolescentes com depressão apresentam-se principalmente irritáveis e instáveis, ao invés de demonstrarem ou queixarem-se de tristeza, podendo ocorrer crises freqüentes de explosão e raiva. Acredita-se que mais de $80 \%$ dos jovens deprimidos apresentam humor irritado (Kazdin \& Marciano, 1998) e ainda perda de energia, apatia e desinteresse importante, retardo psicomotor, sentimentos de desesperança e culpa, perturbações do sono, principalmente hipersônia, alterações de apetite e peso, isolamento e dificuldade de concentração. Outras características próprias desta fase são: prejuízo no desempenho escolar, baixa auto-estima, queixas físicas (dor abdominal, fadiga e cefaléias), idéias e tentativas de suicídio e graves problemas de comportamento, especialmente o uso abusivo de álcool e drogas (Bahls, no prelo B; Brent, 1993; I, 1996; Kashani, Rosenberg \& Reid, 1989; Morgan, 1994; Pataki \& Carlson, 1995; Ryan e cols., 1992; Scivoletto e cols., 1994; Versiani e cols., 2000; Vilela, 1996; Walter, 1996; Ward e cols., 2000). A compreensão do fenômeno da morte em adolescentes acrescenta importância às idéias e tentativas de suicídio nos quadros depressivos desta faixa etária, deixando-os altamente vulneráveis às mesmas.

A distimia na adolescência apresenta sintomas semelhantes aos da depressão maior, com menor intensidade. Entretanto, pela sua natureza crônica, pode causar prejuízos tão ou mais severos do que a depressão maior, justificando a mesma importância e necessidade de tratamento adequado. Os adolescentes distímicos costumam desenvolver menos sintomas melancólicos do que na depressão maior, e $70 \%$ acabam por também apresentar depressão maior no curso de seu quadro clínico, caracterizando a chamada "dupla depressão" (Kovacs, Akiskal, Gatsonis \& Parrone, 1994; Masi, Favilla, Mucci, Poli \& Romano, 2001).

A diferença da manifestação depressiva entre adolescentes do sexo feminino e masculino foi descrita por alguns autores (Baron \& Campbell, 1993; Donnelly, 1995; Morgan, 1994; Sadler, 1991). As garotas relatam mais sintomas subjetivos como sentimentos de tristeza, vazio, tédio e ansiedade; e costumam ter, também, mais preocupação com popularidade, menos satisfação com a aparência, mais conscienciosidade e menos auto-estima. Enquanto os rapazes relatam mais sentimentos de desprezo, desafio e desdém, e demonstram problemas de conduta como: falta às aulas, fugas de casa, violência física, roubos e abuso de subs-tâncias.

Já existem alguns fatores de risco para depressão em adolescentes razoavelmente bem definidos. O mais importante é a presença de depressão em um dos pais, pois sabe-se que a existência de história familiar para depressão aumenta o risco em pelo menos três vezes. Outros fatores importantes de risco são os estressores ambientais como abuso físico e sexual e a perda de um dos pais, irmão ou amigo íntimo (Brage, 1995; Brent, 1993; Lewinsohn, Rohde, Seeley, Klein \& Gotlib, 2000; Shaffi \& Shaffi, 1992). Outros fatores de risco são a presença de conflito familiar, dúvida quanto à orientação sexual, presença de comorbidades, especialmente doenças crônicas, sexo 
feminino, imagem corporal ruim, história de abuso, baixa condição socioeconômica, identificação com grupos minoritários, falta de suporte familiar e poucas habilidades sociais (I, Curatolo \& Friedrich, 2000; Son \& Kirchner, 2000; Ward e cols., 2000).

Deve-se suspeitar de depressão em adolescentes que apresentam problemas escolares (afastamento da escola ou queda importante no rendimento escolar), familiares e legais, alterações significativas de peso corporal, abuso de substâncias (álcool e drogas), comportamento sexual de risco e problemas de conduta (explosões de raiva, brigas freqüentes, vandalismo e roubos). E ainda, estados de humor irritável ou depressivo duradouro e/ou excessivos, períodos prolongados de isolamento ou hostilidade com família e amigos, afastamento de atividades grupais, fugas de casa e presença de queixa física imprecisa, persistente e sem esclarecimento etiológico (Sadler, 1991; Vilela, 1996; Ward e cols., 2000).

\section{Evolução}

A idade média de início da depressão maior na adolescência situa-se aos quatorze anos e nove meses, e a duração do primeiro episódio depressivo é em torno de cinco a nove meses (Bahls, no prelo A; Ward e cols., 2000). A maioria dos autores cita o artigo de Kovacs, Feinberg, Crouse-Novak, Paulauskas e Finkelstein (1984) quando fazem referência a índices de recuperação sobre a depressão maior na adolescência, em que $74 \%$ dos casos apresentaram melhora significativa dentro de um ano, e $92 \%$ se recuperaram em um período de dois anos (Garland, 1994; Garrison e cols., 1997; Harrington, 1992; Kazdin \& Marciano, 1998; Kent e cols., 1997; Larsson e cols., 1991; Olsson \& von Knorring, 1999; Pataki \& Carlson, 1995; Shaffi \& Shaffi, 1992; Walter, 1996). A literatura é unânime em afirmar que, após a recuperação, costuma permanecer algum grau de prejuízo psicossocial, principalmente dificuldades interpessoais crônicas e problemas de conduta; e quanto mais precoce for o aparecimento da patologia, maior tenderá a ser o prejuízo, o que foi confirmado na pesquisa de Rohde e cols. (1994) com adolescentes comunitários onde concluíram que o aparecimento precoce da depressão maior é uma forma mais perniciosa da doença e causa um impacto mais severo do que em adultos. Os dados disponíveis até o momento sugerem um curso provavelmente mais refratário ao tratamento do que quando do início na idade adulta (Martin \& Cohen, 2000).

Adolescentes com depressão possuem um grande risco de recorrência que se estende até a idade adulta, representando uma alta vulnerabilidade para transtornos depressivos no futuro (Bahls, no prelo A).
Segundo artigos de revisão (Birmaher e cols., 1996; Harrington, 1995; Mirza \& Michael, 1996; Pataki \& Carlson, 1995; Walter, 1996), o risco de recorrência da depressão maior na adolescência apresenta taxas entre 33 a $80 \%$ em cinco anos, e é mais freqüente alguns meses após o primeiro episódio. Nos EUA e Inglaterra, em pesquisas longitudinais (Harrington, 1990; Kessler \& Walters, 1998; Kovacs e cols., 1984), foram encontradas taxas de recorrência entre 60 a $74 \%$. Os fatores preditores de recorrência são: início precoce, inúmeros episódios anteriores, gravidade do episódio, presença de sintomas psicóticos, presença de estressores, comorbidade (especialmente distimia) e falta de adesão ao tratamento (Bahls, no prelo A; Versiani e cols., 2000).

Calcula-se que aproximadamente entre $20 \%$ a $40 \%$ dos adolescentes deprimidos irão desenvolver um transtorno bipolar, no período de cinco anos após o início da depressão (Gill e cols., 2000; Ward e cols., 2000). Alguns autores consideram o aparecimento de episódio depressivo maior na infância e na adolescência como indicativo de transtorno bipolar no futuro, entretanto ainda faltam evidências seguras dessa relação (Birmaher e cols., 1996; Olsson \& von Knorring, 1999; Weissman, Wolk, Goldstein, Moreau, Adams e cols., 1999).

\section{Comorbidade}

Costumam ocorrer altas taxas de comorbidade nos quadros depressivos da adolescência, sendo os mais freqüentes os transtornos de ansiedade (30 a 80\%), a distimia (33\%), o abuso de substâncias (20 a 30\%), o transtorno de déficit de atenção com hiperatividade (50\%) e os transtornos de conduta (10 a $80 \%)$, e estes últimos podem persistir após cessar o episódio depressivo (Birmaher e cols., 1996; Goodyer e Cooper, 1993; Harrington, 1995; Harrington e cols., 1994; Herkov \& Myers, 1996; Kent e cols., 1997; Kessler \& Walters, 1998; Nolen-Hoeksema \& Girgus, 1994; Olsson \& von Knorring, 1997b; Pataki \& Carlson, 1995; Rohde e cols., 1994; Walter, 1996). Martin e Cohen (2000) e Scivoletto e cols. (1994) citam que $20 \%$ dos adolescentes deprimidos também apresentam abuso de álcool e drogas, sendo que o início da depressão maior costuma ocorrer quatro a cinco anos antes do início do uso de substâncias (Gill e cols., 2000).

Kessler e Walters (1998), com indivíduos entre quinze a vinte e quatro anos de idade, que fizeram parte do National Comorbidity Survey, nos EUA, encontraram que 76,7\% daqueles com depressão maior e $69,3 \%$ daqueles com distimia apresentavam, pelo menos, um outro transtorno psiquiátrico ao longo da 
vida, e concluíram que a comorbidade para depressão, nesta faixa etária, é mais regra do que exceção. Recentemente, Lewinsohn e cols. (2000) como parte do Oregon Adolescent Depression Project em adolescentes que sofreram diagnóstico de depressão maior antes dos dezenove anos e foram acompanhados até a idade de vinte e três anos, encontraram que $32 \%$ dos casos não tiveram recorrências, $21 \%$ recorreram sem comorbidade associada, $24 \%$ tiveram recorrências e ainda surgiram outras patologias comórbidas e $23 \%$ não apresentaram recorrências mas apresentaram o aparecimento de outras patologias não afetivas. Observaram ainda que naqueles pacientes em que houve o surgimento de outras patologias, a mais comum foi o uso de substâncias representando $77 \%$ dos casos.

A probabilidade de transtornos comórbidos nos quadros de depressão maior na adolescência tende a aumentar na medida da gravidade do quadro depressivo, assim como a sua presença costuma indicar uma evolução mais grave e um prognóstico mais pobre (Bahls, no prelo A; Pataki \& Carlson, 1995; Shaffi \& Shaffi, 1992).

\section{Comportamento Suicida}

O suicídio no adolescente é o fato mais relevante do quadro clínico da depressão. Dentro do espectro do comportamento suicida situam-se as idéias suicidas, as tentativas de suicídio e o suicídio consumado (Bahls, no prelo A). Assim como a depressão, o comportamento suicida entre jovens aparenta estar aumentando nas últimas décadas, e a adolescência destaca-se como o período mais relacionado à morte devido a causas violentas (Emslie, Weinberg, Rush, Adams \& Rintelmann, 1990; Feijó, Salazar, Bozko, Bozko, Lindiago e cols., 1996; Gunnell, 2000; Harrington, 1995; Lamb \& Pusker, 1991; Moreira, 1996; Patten, Gillin, Farkas, Gilpin, Beray e cols., 1997; Prosser \& McArdle, 1996; Roberts \& Chen, 1995; Weissman e cols., 1999).

Entre os anos de 1980 e 1990, na Inglaterra e País de Gales houve um aumento nas taxas de suicídio de $78 \%$ entre jovens de quatorze a vinte e quatro anos de idade (Scivoletto e cols., 1994). Atualmente o suicídio é a segunda causa de morte entre jovens de quinze a vinte e quatro anos de idade, tanto nos EUA, conforme o National Center for Health Statistics (Lamb \& Pusker, 1991) de 1986, quanto na Inglaterra, segundo o estudo Office of Population Census and Surveys (Harrington, Bredenkamp, Groothves, Rutter, Fudge e cols., 1994) de 1990; e o índice de suicídio na população geral, nesta faixa etária, é de $0,01 \%$ (Hurry, 2000). O suicídio é uma das principais causas de morte entre os jovens, e aproximadamente entre um terço a dois terços ocorre em adolescentes clinicamente deprimidos, sendo, portanto, a principal causa de suicídio entre jovens.

Adolescentes apresentam taxas de ideação suicida que variam entre 23 a $27 \%$, e $3,5 \%$ relatam pelo menos uma tentativa de suicídio (Ward e cols., 2000). A ideação suicida é comum em adolescentes, e as tentativas de suicídio e o suicídio em si aumentam com a idade, especialmente após a puberdade. Mais de 25\% dos estudantes do ensino médio (Brent, 1993) apresentaram ideação suicida em levantamentos comunitários. Segundo o United States Youth Risk Survey (1990), 4\% dos estudantes do ensino médio apresentaram uma tentativa de suicídio nos doze meses anteriores, e $8 \%$ já tiveram uma tentativa de suicídio anterior, na vida. O suicídio é três a quatro vezes mais comum em rapazes do que em garotas, enquanto que as tentativas de suicídio são duas a cinco vezes mais comuns nas garotas (Bahls, no prelo A; Brent, 1993; Feijó, Raupp \& John, 1999; Hurry, 2000; Lippi, Pereira, Soares \& Camargos Jr, 1990; Pataki \& Carlson, 1995; Sadler, 1991; Scivoletto e cols., 1994). Especificamente entre adolescentes deprimidos o comportamento suicida alcança índices alarmantes como observado por Weismman e cols. (1999) nos EUA, que, em pesquisa de acompanhamento, em um período entre dez e quinze anos, com adolescentes deprimidos, encontraram uma taxa de tentativas de suicídio de $50,7 \%$ e de $7,7 \%$ de suicídios.

O comportamento suicida na adolescência apresenta alguns fatores conhecidos de risco: idade, sexo masculino, presença de tentativas anteriores, história familiar de transtornos psiquiátricos (especialmente com tentativa de suicídio e/ou suicídio), ausência de apoio familiar, presença de arma de fogo em casa, orientação sexual minoritária, doença física grave e/ou crônica, presença de depressão e comorbidade com transtornos de conduta e abuso de substâncias, sendo que o risco de comportamento suicida em adolescentes deprimidos é três vezes maior na presença destas patologias comórbidas (Feijó e cols., 1996; Gunnell, 2000; Lippi e cols., 1990; Miranda \& Queiroz, 1991; Mirza \& Michael, 1996; Pataki \& Carlson, 1995; Serfaty, Andrade, D’Aquila, Boffi-Boggero, Masaútis e cols., 1996; Ward e cols., 2000). Considera-se a existência de tentativa de suicídio prévia o melhor dos preditores, pois, em torno de $25 \%$ dos adolescentes com tentativa de suicídio e 25 a $40 \%$ dos jovens que se suicidam já tiveram, pelo menos, uma tentativa de suicídio prévia. E estima-se que até $11 \%$ dos adolescentes que fazem tentativa de suicídio através 
de intoxicação irão se suicidar nos próximos anos (Hurry, 2000; Reyes-Ticas, 1995; Ward e cols., 2000). Pelo menos $50 \%$ dos adolescentes que cometem suicídio fizeram ameaças ou tentativas no passado, e o risco de repetir uma tentativa é maior nos três primeiros meses após uma tentativa de suicídio (Brent, 1993). O sentimento de desesperança também está fortemente associado ao comportamento suicida e prediz futuras tentativas (Besseghini, 1997; Feijó e cols., 1997; Ivarsson \& Gilberg, 1997; Lamb \& Pusker, 1991; Pfeffer, 1992, Reyes-Ticas, 1995; Scivoletto e cols., 1994).

Perdas, crises interpessoais com família ou amigos, estressores psicossociais, abuso físico e sexual, problemas legais ou disciplinares e a exposição ao suicídio de amigos, familiares ou através da mídia são precipitantes do comportamento suicida (Besseghini, 1997; Brent, 1993; Feijó e cols., 1999; Hurry, 2000; Pataki \& Carlson, 1995; Pfeffer, 1992; Scivoletto e cols., 1994).

Adolescentes deprimidos, portanto, apresentam claramente a necessidade cuidadosa de acompanhamento especializado. Os clínicos devem estar alertas e considerarem cuidadosamente qualquer manifestação de ideação ou comportamento suicida em jovens, mesmo naqueles que não aparentam psicopatologia. A melhor maneira de prevenir o suicídio é a detecção precoce e o tratamento das patologias psiquiátricas que o predispõe.

\section{Conclusões}

$\mathrm{O}$ estudo dos transtornos depressivos na adolescência se reveste da maior importância na medida que representa não só um grupo de patologias

\section{Referências}

American Psychiatric Association. (1994). Diagnostic and statistical manual of mental disorders. DSM-IV, 4th. ed. Washington, DC: American Psychiatric Association.

Angold, A. \& Costello, E. J. (1995). The epidemiology of depression in children and adolescents. Em I. M. Goodyer (Org.), The depressed child and adolescent: Developmental and clinical perspectives (p. 127-147). Cambridge University Press: Cambridge.

Bahls, S. C. (1999). Depressão: Uma breve revisão dos fundamentos biológicos e cognitivos. Interação, 3, 49-60.

Bahls, S. C. (2002). Epidemiology of depressive symptoms in adolescents of a public school in Curitiba, Brazil. Revista Brasileira de Psiquiatria, 24(2), 63-67.

Bahls, S. C. (no prelo A). Aspectos clínicos da depressão na infância e na adolescência. Jornal de Pediatria.

Bahls, S. C. (no prelo B). Medicamentos antidepressivos e insônia. Revista Temas em Psiquiatria. com altos e crescentes índices na população, como também em muitos casos conduz a um trágico desfecho através do suicídio. Essas considerações projetam a doença depressiva em adolescentes como um dos principais problemas de saúde pública. Sabese atualmente, que os transtornos depressivos em adultos são pouco diag-nosticados, e mesmo nos casos identificados, grande parte deles não recebe tratamento adequado. Este panorama assume proporções ainda mais preocupantes quando se trata de adolescentes, pois o diagnóstico nesta faixa etária é mais difícil, e pesquisas em países desenvolvidos mostram que os adolescentes identificados em pesquisas comunitárias como portadores de depressão maior raramente estavam diagnosticados ou em tratamento (Donnelly, 1995; Feijó e cols., 1997; Goodyear \& Cooper, 1993; Harrington e cols., 1994; Martin \& Cohen, 2000; Roberts e cols., 1995). A esta conjuntura deve-se associar as dificuldades da sintomatologia da depressão juvenil que apresenta uma patoplastia própria desta fase do desenvolvimento, trazendo características clínicas específicas, como examinado no âmbito do quadro clínico deste artigo. Também o alto índice de comorbidades acrescenta mais desafios quanto ao campo diagnóstico em adolescentes, o que deve contribuir para alertar os profissionais envolvidos no trato com esta comum condição clínica. O estudo específico da depressão na adolescência é recente e sofre por esta condição ainda de muitas imprecisões, que esperamos, em breve, sejam esclarecidas como fruto dos estudos que estão sendo realizados, trazendo benefícios e avanços que permitam uma melhor compreensão e tratamento desta patologia.

Baptista, C. A. \& Golfeto, J. H. (2000). Prevalência de depressão em escolares de 7 a 14 anos. Revista de Psiquiatria Clínica, 27(5).

Baron, P. \& Campbell, T. L. (1993). Gender differences in the expression of depressive symptoms in middle adolescents: an extension of early findings. Adolescence, 28(112), 903-911.

Berganza, C. E. \& Aguilar, G. (1992). Depression in guatemalan adolescents. Adolescence, 27(108), 771-782.

Besseghini, V. H. (1997). Depression and Suicide in Children and Adolescents. Annals New York Academy of Sciences, 816, 9498.

Bhatara, V. S. (1992). Early detection of adolescent mood disorders. South Dakota Journal Medicine, 45 (3), 75-78.

Birmaher, B.; Ryan, N. D.; Williamson, D. E.; Brent, D. A.; Kaufman, J.; Dahl, R. E.; Perel, J. \& Nelson, B. (1996). Childhood and adolescent depression: A review of the past 10 years. Part I. Journal of American Academic Child Adolesencec Psychiatry, 35(11), 1427-1439.

Brage, D. G. (1995). Adolescent depression: A review of the literature. Archives of Psychiatric Nursing, IX(1), 45-55. 
Brent, D. A. (1993). Depression and suicide in children and adolescents. Pediatrics in Review, 14(10), 380-388.

Busse, S. R. (1996). Histórico e conceito dos transtornos afetivos na infância e na adolescência. Em A. Assumpção Jr. (Org.) Transtornos afetivos da infância e adolescência (p. 11-17). São Paulo: Lemos.

Chartier, G. M. \& Lassen, M. K. (1994). Adolescent depression: children's depression inventory norms, suicidal ideation, and (weak) gender effects. Adolescence, 29(116), 857-864.

Cross-National Collaborative Group. (1992). The changing rate of major depression: Cross-national comparisons. Journal American Medical Association, 268, 3098-3105.

Donnelly, M. (1995). Depression among adolescents in Northern Ireland. Adolescence, 30(118), 339-350.

Emslie, G. J.; Weinberg, W. A.; Rush, A. J.; Adams, R. M. \& Rintelmann, J. W. (1990). Depressive symptoms by self-report in adolescence: Phase I of the development of a questionnaire for depression by self-report. Journal of Child Neurology, 5, 114-121.

Feijó, R. B.; Salazar, C. C.; Bozko, M. P.; Bozko, M.; Candiago, R. H.; Ávila, S.; Rocha, T. S. \& Chaves, M. L. F. (1996). O adolescente com tentativa de suicídio: características de uma amostra de 13 a 20 anos atendida em emergência médica. Jornal Brasileiro de Psiquiatria, 45(11), 657-664.

Feijó, R. B.; Saueressig. M.; Salazar, C. \& Chaves, M. L. F. (1997). Mental health screening by self-report questionnaire among community adolescents in southern Brazil. Journal of Adolescent Health, 20, 232-237.

Feijó, R. B.; Raupp, A. P. G. \& John, A. B. (1999). Eventos estressores de vida e sua relação com tentativas de suicídio em adolescentes. Jornal Brasileiro de Psiquiatria, 48(4), 151-157.

Garland, J. (1994). Adolescent depression Part I. Diagnosis. Canadian Family Physician, 40, 1583-1587.

Garrison, C. Z.; Addy, C. L.; Jackson, K. L.; McKeown, R. E. \& Waller, J. L. (1997). Incidence of major depressive disorder and dysthymia in young adolescents. Journal of the American Academy of Child \& Adolescent Psychiatry, 36(4), 458-465.

Gill, S. K.; Coffey, B. J. \& Park, K. S. (2000). Depressão na infância e na adolescência: Manifestações clínicas, patogênese e tratamento. Em B. Lafer, O. P. Almeida, R. Fráguas Jr R \& E. C. Miguel (Orgs.), Depressão no ciclo da vida (p. 232-245). Porto Alegre: Artes Médicas.

Goodyer, I. M. (1996). Physical symptoms and depressive disorder in childhood and adolescence. Journal of Psychosomatic Research, 41(5), 405-408.

Goodyer, I. \& Cooper, P. J. (1993). A community study of depression in adolescent Girls II: The clinical features of identified disorder. Brazilian Journal of Psychiatry, 163, 374380 .

Gunnell, D. J. (2000). The epidemiology of suicide. International Review of Psychiatry, 12(1), 21-26.

Harrington, R. (1992). The natural history and treatment of child and adolescent Affective disorders. Journal of Child Psychology and Psychiatry, 33(8), 1287-1302.

Harrington, R. (1995). Depressive disorder in adolescence. Journal of British Paediatric Association, 72(3), 193-195.

Harrington, R.; Fudge, H.; Rutter, M.; Pickles, A. \& Hill, J. (1990). Adult outcomes of childhood and adolescent depression: I. Psychiatric status. Archives of General Psychiatry, 47, 465473.

Harrington, R.; Bredenkamp, D.; Groothues, C.; Rutter, M. Fudge, H. \& Pickles, A. (1994). Adult outcomes of childhood and adolescent depression. III Links with suicidal behaviors. Journal of Child Psychology and Psychiatry, 35(7), 1309-1319.

Herkov, M. J. \& Myers, W. C. (1996). MMPI profiles of depressed adolescents with and without conduct disorder. Journal of Clinical Psychology, 52(6), 705-710.

Hirschfeld, R. M. A. (1998). Long-term nature of depression. Depression and anxiety, 7 (suppl 1), 1-4.

Hurry, J. (2000). Deliberate self-harm in children and adolescents. International Review of Psychiatry, 12(1), 31-36.

I, L. F. (1996). Epidemiologia e fatores clínicos dos transtornos afetivos da infância e adolescência. Em A. Assumpção Jr. (Org.), Transtornos afetivos da infância e adolescência (p. 1928). São Paulo: Lemos.

I, L. F.; Silva, S. M. P. B. R. \& Machado, L. T. (1998). Uso de Citalopram em meninos deprimidos de 10 a 13 anos: relato de 4 casos. Psiquiatria Biológica, 6(3), 133-138.

I, L. F.; Curatolo, E. \& Friedrich, S. (2000). Transtornos afetivos. Revista Brasileira de Psiquiatria (suppl 2); 22, 24-27.

Ivarsson, T. \& Gillberg, C. (1997). Depressice symptoms in Swedish adolescents: Normative data using the Birleson Depression Self-Rating Scale (DSRS). Journal of Affective Disorders, 42, 59-68.

Joyce, P. R.; Oakley-Browne, M. A.; Wells, J. F.; Bushnell, J. A. \& Hornblow, A. R. (1990). Birth Cohort trends in major depression: Increasing rates and earlier onset in New Zealand. Journal of Affective Disorders 18, 83-89.

Judd, L. L. (1995). Mood disorders in the general population represent na important and worldwide public health problem. International Clinical Psychopharmacol, 10 (suppl 4), 5-10.

Kashani, J. H.; Rosenberg, T. \& Reid, J. (1989). Developmental perspectives in child and adolescent depressive symptoms in a community sample. American Journal of Psychiatry, 146, 871875 .

Kazdin, A. E. \& Marciano, P. L. (1998). Childhood and adolescent depression. Em E. Mash \& R. Barkley (Orgs.), Treatment of childhood disorders $\left(2^{\mathrm{a}}\right.$ ed.) New York: The Guilford Press.

Kent, L.; Vostanis, P. \& Feehan, C. (1997). Detection of major and minor depression in children and adolescents: Evaluation of the mood and feelings questionnaire. Jounral of Child Psychology and Psychiatry, 38(5), 565-573.

Kessler, R. C. \& Walters, E. E. (1998). Epidemiology of DSM-III$\mathrm{R}$ major depression and minor depression among adolescents and young adults in the national comorbidity survey. Depression and Anxiety, 7, 3-14.

Klerman, G. L. \& Weissman, M. M. (1989). Increasing rates of depression. JAMA. 261, 2.229-2.235.

Klerman, G. L.; Lavori, P. W.; Rice, J.; Reich, T.; Endicott, J. \& Andreasen, N. C. (1985). Birth-cohort trends in rates of major depressive disorder among relatives of patients with affective disorder. Archives of General Psychiatry, 42, 689-693.

Kovacs, M.; Feinberg, T. L.; Crouse-Novak, M. A.; Paulauskas, S. L. \& Finkelstein, R. (1984). Depressive disorders in childhood: I. A longitudinal prospective study of characteristics and recovery. Archives of General Psychiatry, 41, 229-237.

Kovacs, M.; Akiskal, S.; Gatsonis, C. \& Parrone, P. L. (1994). Childhood-onset dysthymic disorder. Archives of General Psychiatry, 51, 365-374.

Lamb, J. \& Pusker, K. R. (1991). School-based adolescent mental health project survey of depression, suicidal ideation, and anger. JCPN, 4(3), 101-104.

Larsson, B.; Melin, L.; Breitholtz, E. \& Andersson, G. (1991). Short-term stability of depressive symptoms and suicide 
attempts in Swedish adolescents. Acta Psyciatrica Scandinavica, 83, 385-390.

Lewinsohn, P. M.; Clarke, G. N.; Seeley, J. R. \& Rohde, P. (1994). Major depression in community adolescents: Age at onset, episode duration, and time to recurrence. American Journal of Academy of Child \& Adolescent Psychiatry, 33(6), 809-818.

Lewinsohn, P. M.; Rohde, P.; Seeley, J. R.; Klein, D. N. \& Gotlib, I. H. (2000). Natural course of adolescent major depressive disorder in a community sample: Predictors of recorrence in young adults. American Journal of Psychiatry, 157(10), 1.5841.591 .

Lippi, J. R. S.; Pereira, I. M.; Soares, K. V. S. \& Camargos Jr, W. (1990). Suicídio na infância e adolescência. Jornal Brasileiro de Psiquiatria, 39(4), 167-174.

Martin, A. \& Cohen, D. J. (2000). Adolescent depression: Window of (missed?) opportunity - Editorial. American Journal of Psychiatry, 157(10), 1549-1.551.

Masi, G.; Favilla, L.; Mucci, M.; Poli, P. \& Romano, R. (2001). Depressive symptoms in children and adolescents with dysthymic disorder. Psychopathology, 34, 29-35.

Miranda, P. S. C. \& Queiroz, E. A. (1991). Pensamento suicida e tentativa de suicídio entre estudantes de medicina. Revista $A B P$ $A P A L, 13(4), 157-160$

Mirza, K. A. H. \& Michael, A. (1996). Major depression in children and adolescents. Br J Hospital Medicine, 55(1-2), 5761.

Moreira, M. S. (1996). A psicose maníaco-depressiva na infância e na adolescência. Jornal Braeileiro des Psiquiatria, 45(2), 6974.

Morgan, I. S. (1994). Recognizing depression in the adolescent. $M C N, 19,148-155$.

Murray, C. J. L. \& Lopez, A. D. (1996). The global burden of disease and injury series. The global burden of disease (Summary). Harvard School of Public Health: Harvard University Press.

Nolen-Hoeksema, S. \& Girgus, J. S. (1994). The emergence of gender differences in depression during adolescence. Psychological Bulletin, 115(3), 424-443.

Olsson, G. \& von Knorring, A-L. (1997a). Beck's depression inventory as a screening instrument for adolescent depression in Sweden: gender differences. Acta Psychiatrica Scandinavica, $95,277-282$

Olsson, G. \& von Knorring, A-L. (1997b). Depression among Swedish adolescents measured by the self-rating Scale Center for Epidemiology Studies - Depression Child (CES-DC). European Child \& Adolescent Psychiatry, 6, 81-87.

Olsson, G. \& von Knorring, A-L. (1999). Adolescent depression: prevalence in Swedish high-school students. Acta Psychiatrica Scandinavica, 99, 324-331.

Organização Mundial da Saúde. (1993). Classificação de transtornos mentais e de comportamento da CID-10. Descrições clínicas e diretrizes diagnósticas. Porto Alegre. Artes Médicas.

Pataki, C. S. \& Carlson, G. A. (1995). Childhood and adolescent depression: A review. Harvard Review of Psychiatry, 3(3), 140151.

Patten, C. A.; Gillin, J. C.; Farkas, A. J.; Gilpin, E. A.; Berry, C. C. \& Pierce, J. P. (1997). Depressive symptons in California adolescents: Family structure and parental support. Journal of Adolescent Health, 20, 271-278.

Pfeffer, C.R. (1992). Relationship between depression and suicidal behavior. Em M. Shaffi \& S. L. Shaffi (Orgs.), Clinical guide to depression in children and adolescents (p. 115-126). Washington: American Psychiatric Press.

Prosser, J. \& McArdle, P. (1996). The changing mental health of children and adolescents: evidence for a deterioration? Psychological Medicine, 26, 715-725.

Reyes-Ticas, J. A. (1995). Suicidios y intentos de suicidio en Honduras. Psiquiatria Biológica, 3, 57-64.

Roberts, R. E. \& Chen, Y-W. (1995). Depressive symptoms and suicidal ideation among mexican-origin and anglo adolescents. Journal of American Academy Child \& Adolesentc Psychiatry, 34(1), 81-90.

Roberts, R. E.; Lewinsohn, P. M. \& Seeley, J. R. (1995). Symptoms of DSM-III-R major depression in adolescence: Evidence from na epidemiological survey. Journal of American Academy of Child \& Adolesent Psychiatry, 34(12), 1608-1617.

Rohde, P.; Lewinsohn, P. M. \& Seeley, J. R. (1994). Are adolescents changed by na episode of major depression? Journal of American Academy of Child \& Adolesentc Psychiatry, 33(9), 1289-1298.

Ryan, N. D.; Williamson, D. E.; Iyengar, S.; Orvaschel, H.; Reich, T.; Dahl, R. E. \& Puig-Antich, J. (1992). A secular increase in child and adolescent onset affective disorder. Journal of American Academy of Child \& Adolescent Psychiatry, 31(4), 600-605.

Sadler, L. S. (1991). Depression in adolescents. Context, manifestations, and clinical management. Nursing Clinics of North America, 26(3), 559-572.

Scivoletto, S.; Nicastri, S. \& Zilberman, M. L. (1994). Transtorno depressivo na adolescência: diagnóstico e tratamento. Revista Brasileira de Medicina, 51(9), 1211-1228.

Serfaty, E.; Andrade, J. H.; D’Aquila, H.; Boffi-Boggero, H. J.; Masaútis, A. E. \& Foglia, V. L. (1996). Depression and risk factors in 18-year-old males in 5 districts of the Argentina Republic in 1992. Psiquiatria Biológica, 4(4), 207-212.

Shaffi, M. \& Shaffi, S. L. (1992). Clinical manifestations and developmental psychopathology of depression. Em M. Shaffi \& S. L. Shaffi (Orgs.), Clinical guide to depression in children and adolescents (p. 3-42). Washington: American Psychiatric Press.

Son, S. E. \& Kirchner, J. T. (2000). Depression in children and adolescents. Am Fam Physician, 62, 2297-2308.

Souza, P. R. (1984). Depressão endógena em criança de oito anos - relato de caso. Revista da Associação Brasileira de Psiquiatria, 6(21), 67-69.

Vilela, J. E. M. (1996). Sintomas depressivos das depressões. Psiquitria Biológica, 4, 95-99.

Versiani, M.; Reis, R. \& Figueira, I. (2000). Diagnóstico do transtorno depressivo na infância e adolescência. $J$ Bras Psiquiatria, 49(10-12), 367-382.

Walter, G. (1996). Depression in adolescence. Australian Family Physician, 25(10), 1575-1582.

Ward, R. K.; Eyler, A. E. \& Makris, G. R. (2000). Evaluation and management of depressive illness in adolescence. Clinics in Family Practice. 2.

Weissman, M. M.; Wolk, S.; Goldstein, R. B.; Moreau, D.; Adams, P.; Greenwald, S.; Klier, C. M.; Ryan, N. D.; Dahl, R. E. \& Wickramaratne, P. (1999). Adolescentes deprimidos: Como ficam quando adultos. Jama Brasil, 3(9), 2429-2435.

World Health Organization. (1992). The ICD-10 classification of mental and behavioral disorders. Clinical descriptions and diagnostic guidelines. Genebra: World Health Organization.

York, A. \& Hill, P. (1997). Depression and physical illness in childhood and adolescence. Em M. M. Robertson \& C. L. E. 
Katona (Org.), Depression and physical illness (p. 145-168). West Sussex. Wiley.

Recebido: 12/12/2001

Revisado: 05/05/2002 Aceito: 20/05/2002 
Sobre os autores

Saint-Clair Bahls: Pós-graduado em Psiquiatria Clínica UFRS; Mestre em Psicologia da Infância e Adolescência UFPR; Prof. Departamento Psicologia UFPR. E-mail: scbahls@uol.com.br

Flávia Rocha Campos Bahls: Psicóloga clínica; Mestranda em Psicologia da Infância e da Adolescência UFPR. 